\title{
MILNOR-HAMM SPHERE FIBRATIONS AND THE EQUIVALENCE PROBLEM
}

\author{
RAIMUNDO N. ARAÚJO DOS SANTOS, MAICO F. RIBEIRO, AND MIHAI TIBĂR
}

\begin{abstract}
We introduce the sphere fibration for real map germs with radial discriminant and we address the problem of its equivalence with the Milnor-Hamm tube fibration. Under natural conditions, we prove the existence of open book structures with singularities and solve the equivalence problem.
\end{abstract}

\section{INTRODUCTION}

Let $G:\left(\mathbb{R}^{m}, 0\right) \rightarrow\left(\mathbb{R}^{p}, 0\right), p \geq 2$, be a non-constant real analytic map germ. Under the condition that $G$ has isolated singularity at $0 \in \mathbb{R}^{m}$, it was shown by Milnor [Mi] that there exists a tube fibration and a sphere fibration. Together they contribute to the definition of a higher open book structure as explained in [AT1, AT2, ACT] in the more general case when the singular set $\operatorname{Sing} G$ is non-isolated but still included in the central fibre $G^{-1}(0)$. Milnor [Mi] construction of a sphere fibration by the method of blowing away the tube fibration holds under certain conditions (see the discussion in [AT2, §2]), providing a topological equivalence between the empty tube fibration and the sphere fibration.

In case of holomorphic functions $f:\left(\mathbb{C}^{n}, 0\right) \rightarrow(\mathbb{C}, 0)$, Milnor [Mi] proved that the sphere fibration is induced by the special map $f /\|f\|$. However this particular construction does not extend to real map germs, as already suggested by Milnor in [Mi, §11]. There have been several successful tries to add up supplementary conditions so that the map $G /\|G\|$ defines a fibration, e.g. [Ja, RSV, RA, AT1, AT2, CSS, Ar] etc. In case this fibration exists, there remains the question if it is equivalent to the empty tube fibration. This seems not to have been solved even in the case when $G$ has isolated singularity ${ }^{1}$, the setting in which Milnor [Mi, §9] had already given several related results. We formulate this problem here as the Equivalence Conjecture 4.4.

Recently one started to enrich this landscape by treating the case of a positive dimensional discriminant $\operatorname{Disc} G$. As one can easily see, the classical case Disc $G=\{0\}$ remains a very special situation, which for instance never happens in case of maps $\left(\mathbb{C}^{n}, 0\right) \rightarrow\left(\mathbb{C}^{p}, 0\right)$ with $p>1$ defining isolated complete intersection singularities.

2010 Mathematics Subject Classification. 32S55, 14D06, 58K05, 57R45, 14P10, 58K15, 32S60.

Key words and phrases. singularities of real analytic maps, Milnor fibrations, mixed functions.

The authors acknowledge the support of USP-COFECUB Uc Ma 163-17. MT acknowledges the support of the Labex CEMPI (ANR-11-LABX-0007-01). RNAS acknowledges the Fapesp grant 2017/20455-3 and the CNPq grant 313780/2017-0.

${ }^{1}$ The proof claimed in [CSS] seems to be incomplete as pointed out in [Han] and [Ri]. 
The tube fibration for positive dimensional discriminant, predicted in [ACT], has been introduced recently in [ART] under the name Milnor-Hamm tube fibration, and several new classes of singular map germs with such fibration have been presented. This means that over each connected component of the complement of Disc $G$ there is a well-defined locally trivial fibration, and there are finitely many such components ${ }^{2}$.

According to Milnor's program [Mi] detailed in [AT2], there are two more steps in order to define open book structures with singularities. Our paper is devoted to this task in the most reasonable setting of a radial discriminant. We introduce the Milnor-Hamm sphere fibration, we give natural sufficient conditions such that this exists, and we exhibit several such classes of singular maps.

We then state the problem of the equivalence with the corresponding Milnor-Hamm empty tube fibration and we show how to solve it in our general setting under natural supplementary conditions.

Several conditions for the existence of the Milnor vector field are presented in [AR], and further details can be found in [Ri].

\section{The Singular tube fibration}

2.1. Nice map germs. Given a non-constant analytic map germ $G:\left(\mathbb{R}^{m}, 0\right) \rightarrow\left(\mathbb{R}^{p}, 0\right)$, $m \geq p>0$, the set germ $\operatorname{Sing} G$ is well defined on the source space but the images $G(\operatorname{Sing} G)$ and $\operatorname{Im} G$ are in general not well-defined as germs of subanalytic sets, see [ART, JT1]. If they are, then we say that $G$ is a nice map germ.

A simple example of a non-nice map germ is $(x, x y):\left(\mathbb{C}^{2}, 0\right) \rightarrow\left(\mathbb{C}^{2}, 0\right)$, for which $\operatorname{Im} G$ is not a germ. Under these notations, one has the following results about the existence of nice map germs ${ }^{3}$ :

[ART, Lemma 2.4] If Sing $G \cap G^{-1}(0) \subsetneq G^{-1}(0)$ then $\operatorname{Im} G$ contains an open neighbourhood of the origin.

[ART, Theorem 2.7] Let $f, g:\left(\mathbb{C}^{n}, 0\right) \rightarrow(\mathbb{C}, 0)$ be complex polynomials with no common factor of order $\geq 1$. Then $f \bar{g}:\left(\mathbb{C}^{n}, 0\right) \rightarrow(\mathbb{C}, 0)$ is a nice map germ.

As introduced in [ART], we shall call discriminant of a nice map germ $G$ the following set:

$$
\operatorname{Disc} G:=\overline{G(\operatorname{Sing} G)} \cup \partial \overline{\operatorname{Im} G}
$$

where the boundary $\partial \overline{\operatorname{Im} G}:=\overline{\operatorname{Im} G} \backslash \operatorname{int}(\operatorname{Im} G)$ is a closed subanalytic proper subset of $\mathbb{R}^{p}$ and well-defined as a set germ, where $\operatorname{int} A:=\AA$ denotes the $p$-dimensional interior of a semianalytic set $A \subset \mathbb{R}^{p}$ (hence it is empty whenever $\operatorname{dim} A<p$ ), and $\bar{A}$ denotes the closure of it.

It follows from the definition that Disc $G$ is a closed subanalytic set of dimension strictly less than $p$, well-defined as a germ.

\footnotetext{
${ }^{2}$ A subanalytic set has locally finitely many connected components, see e.g. [BM].

${ }^{3}$ For more refined results concerning nice map germs $(f, g)$ and $f \bar{g}$ in $n$ variables, one may consult the recent works [JT1, JT2].
} 


\subsection{The Milnor-Hamm fibration.}

Definition 2.1. [ART, Definition 2.1] Let $G:\left(\mathbb{R}^{m}, 0\right) \rightarrow\left(\mathbb{R}^{p}, 0\right)$ be a non-constant analytic nice map germ. We say that $G$ has a Milnor-Hamm tube fibration if for any $\varepsilon>0$ small enough, there exists $0<\eta \ll \varepsilon$ such that the restriction:

$$
G_{\mid}: B_{\varepsilon}^{m} \cap G^{-1}\left(B_{\eta}^{p} \backslash \operatorname{Disc} G\right) \rightarrow B_{\eta}^{p} \backslash \operatorname{Disc} G
$$

is a locally trivial smooth fibration which is independent, up to diffeomorphisms, of the choices of small enough $\varepsilon$ and $\eta$.

We then also say that the restriction of (2) over a small enough sphere (still denoted by $S_{\eta}^{p}$ but keeping in mind that the radius is slightly smaller than the $\eta$ in (2)):

$$
G_{\mid}: B_{\varepsilon}^{m} \cap G^{-1}\left(S_{\eta}^{p} \backslash \operatorname{Disc} G\right) \rightarrow S_{\eta}^{p} \backslash \operatorname{Disc} G
$$

is a Milnor-Hamm empty tube fibration.

One defines in [ART] a more general notion of stratified tube fibration called singular Milnor tube fibration by considering in addition all singular fibres over the stratified discriminant. In all cases, the tube fibration is a collection of finitely many fibrations over path-connected subanalytic sets.

2.3. $\rho$-regularity of map germs. Let $U \subset \mathbb{R}^{m}$ be an open set, $0 \in U$, and let $\rho: U \rightarrow$ $\mathbb{R}_{\geq 0}$ be the square of the Euclidean distance to 0 . We recall the following definition from [ART]:

Definition 2.2. Let $G:\left(\mathbb{R}^{m}, 0\right) \rightarrow\left(\mathbb{R}^{p}, 0\right)$ be a non-constant analytic nice map germ. The set germ at the origin:

$$
M(G):=\overline{\left\{x \in U \mid \rho \oiint_{x} G\right\}}
$$

is called the set of $\rho$-nonregular points of $G$, or the Milnor set of $G$.

The following inclusion of set germs at the origin will play an important role, where $V_{G}:=G^{-1}(0)$ :

$$
\overline{M(G) \backslash G^{-1}(\operatorname{Disc}(G))} \cap V_{G} \subseteq\{0\} .
$$

Condition (4) is a direct extension of the condition used in [AT1, AT2, Ma, ACT] in case $\operatorname{Disc}(G)=\{0\}$; it was shown that it is implied by the Thom regularity condition, in loc.cit. and several other papers. The reciprocal is however not true, counterexamples are provided in [Ti, ACT], see also [Oka3, PT1].

Condition (4) enables the following existence result proved in [ART, Lemma 3.3]:

Let $G:\left(\mathbb{R}^{m}, 0\right) \rightarrow\left(\mathbb{R}^{p}, 0\right)$ be a non-constant analytic nice map germ. If $G$ satisfies condition (4), then $G$ has a Milnor-Hamm tube fibration (2).

\section{The Milnor-Hamm sphere fibration}

We introduce a natural condition under which one may define sphere fibrations whenever $G$ is a nice map germ and $\operatorname{Disc} G$ is positive dimensional. 
Definition 3.1. Let $G:\left(\mathbb{R}^{m}, 0\right) \rightarrow\left(\mathbb{R}^{p}, 0\right)$ be a real analytic nice map germ. We say that its discriminant Disc $G$ is radial if it is the germ of a real cone with vertex at the origin (i.e. a union of real half-lines at the origin), or just the origin.

ExAmple 3.2. Let $f, g:\left(\mathbb{C}^{n}, 0\right) \rightarrow(\mathbb{C}, 0)$ be holomorphic function germs such that $f$ and $g$ do not have any common factor of order $>0$. Then $f \bar{g}:\left(\mathbb{C}^{n}, 0\right) \rightarrow(\mathbb{C}, 0)$ is a nice map germ by [ART, Theorem 2.7]. If Disc $(f, g)$ is radial, then obviously Disc $f \bar{g}$ is radial, e.g. by [PT1, Lemma 2.4].

EXAMPLE 3.3. Let $f:\left(\mathbb{R}^{m}, 0\right) \rightarrow\left(\mathbb{R}^{p}, 0\right)$ be a real analytic nice map germ, and let $g$ : $(\mathbb{R}, 0) \rightarrow(\mathbb{R}, 0)$ be an analytic invertible germ, such that $f$ and $g$ are in separate variables. Then the map germ $G:=(f, g):\left(\mathbb{R}^{m} \times \mathbb{R}, 0\right) \rightarrow\left(\mathbb{R}^{p} \times \mathbb{R}, 0\right)$ has Sing $G=\operatorname{Sing} f \times \mathbb{R}$ and it is nice. If moreover Disc $f$ is radial, then $\operatorname{Disc} G$ is radial.

Let $G: U \rightarrow \mathbb{R}^{p}$ be a representative of the nice map germ $G$ in some open set $U \ni 0$. We consider the map

$$
\Psi_{G}:=\frac{G}{\|G\|}: U \backslash V_{G} \rightarrow S_{1}^{p-1} .
$$

If $G$ is a nice map germ such that Disc $G$ is radial, then it follows from the definitions that the restriction:

$$
\Psi_{G \mid}: S_{\varepsilon}^{m-1} \backslash G^{-1}(\operatorname{Disc} G) \rightarrow S_{1}^{p-1} \backslash \operatorname{Disc} G
$$

is well defined for any $\varepsilon>0$ small enough.

Definition 3.4. We say that the nice map germ $G:\left(\mathbb{R}^{m}, 0\right) \rightarrow\left(\mathbb{R}^{p}, 0\right)$ with radial discriminant has a Milnor-Hamm sphere fibration if the restriction map (6) is a locally trivial smooth fibration which is independent, up to diffeomorphisms, of the choice of $\varepsilon>0$ provided it is small enough.

Let $M\left(\Psi_{G}\right)$ be the Milnor set of the map (5), i.e. the germ at the origin of the $\rho$ nonregular points of $\Psi_{G}$, cf Definition 2.2. We say that $\Psi_{G}$ is $\rho$-regular if:

$$
M\left(\Psi_{G}\right) \subset G^{-1}(\operatorname{Disc} G) .
$$

REMARK 3.5. The reason for restricting our definition of Milnor-Hamm sphere fibration to radial discriminants is precisely that the restriction (6) of the map $\frac{G}{\|G\|}$ is well-defined if and only if the discriminant is radial. For non-radial discriminants there is a different way to define singular open books without using the map $\frac{G}{\|G\|}$, see Remark 3.7 and Conjecture 3.8.

3.1. Existence of Milnor-Hamm sphere fibrations. The following existence criterion extends the case Disc $G=\{0\}$ considered in [ACT, Theorem 1.3].

Theorem 3.6. Let $G:\left(\mathbb{R}^{m}, 0\right) \rightarrow\left(\mathbb{R}^{p}, 0\right), m>p \geq 2$, be a non-constant analytic nice map germ with radial discriminant, satisfying the condition (4). If $\Psi_{G}$ is $\rho$-regular then $G$ has a Milnor-Hamm sphere fibration.

Proof. The condition (7) controls the topology of the map $\Psi_{G}$ on the complementary of a tubular neighbourhood of $V_{G}$, while the condition (4) controls the behaviour of the map $\Psi_{G}$ close to $V_{G}$. Both conditions are essential, as one can see in many examples. 
Step 1. Under the condition (4), by [ART, Lemma 3.3], the restriction

$$
G_{\mid}: S_{\varepsilon}^{m-1} \cap G^{-1}\left(\bar{B}_{\eta}^{p} \backslash \operatorname{Disc} G\right) \rightarrow \bar{B}_{\eta}^{p} \backslash \operatorname{Disc} G
$$

is a locally trivial fibration for any small enough $0<\eta \ll \varepsilon$. Since Disc $G$ is radial, for $\pi:=s /\|s\|$ we have that

$$
\pi: \bar{B}_{\eta}^{p} \backslash \operatorname{Disc} G \rightarrow S_{1}^{p-1} \backslash \operatorname{Disc} G
$$

is a locally trivial fibration, and the equality $\pi\left(S_{\eta}^{p-1} \cap \operatorname{Disc} G\right)=S_{1}^{p-1} \cap \operatorname{Disc} G$. Composing the maps (8) and (9) one concludes that

$$
\Psi_{G \mid}: S_{\varepsilon}^{m-1} \cap G^{-1}\left(\bar{B}_{\eta}^{p} \backslash \operatorname{Disc} G\right) \rightarrow S_{1}^{p-1} \backslash \operatorname{Disc} G
$$

is a locally trivial fibration, and its restriction to the boundary of the empty tube $S_{\varepsilon}^{m-1} \cap$ $G^{-1}\left(S_{\eta}^{p-1} \backslash \operatorname{Disc} G\right)$ coincides with the following restriction of $G$ :

$$
G_{\mid}: S_{\varepsilon}^{m-1} \cap G^{-1}\left(S_{\eta}^{p-1} \backslash \operatorname{Disc} G\right) \rightarrow S_{\eta}^{p-1} \backslash \operatorname{Disc} G .
$$

More precisely, in our case of a radial discriminant, the bases of the fibrations (3) and (10) can be identified with $\left(\pi_{S_{\eta}^{p-1} \backslash \operatorname{Disc} G}\right)^{-1}: S_{1}^{p-1} \backslash \operatorname{Disc} G \rightarrow S_{\eta}^{p-1} \backslash \operatorname{Disc} G$, which is the multiplication by $\eta$.

Step 2. The condition (7) is equivalent to the fact that the map $\Psi_{G}: S_{\varepsilon}^{m-1} \backslash G^{-1}$ (Disc $\left.G\right) \rightarrow$ $S_{1}^{p-1} \backslash \operatorname{Disc} G$ is a submersion (over its image) for any small enough $\varepsilon$. Consequently, the restriction

$$
\Psi_{G \mid}: S_{\varepsilon}^{m-1} \backslash\left\{G^{-1}(\operatorname{Disc} G) \cup G^{-1}\left(B_{\eta}^{p}\right)\right\} \rightarrow S_{1}^{p-1} \backslash \operatorname{Disc} G
$$

is a submersion. It coincides with the fibration (11) on $S_{\varepsilon}^{m-1} \cap G^{-1}\left(S_{\eta}^{p-1} \backslash \operatorname{Disc}(G)\right)$. Moreover, the map (12) is proper since the restriction $\Psi_{G \mid}: S_{\varepsilon}^{m-1} \backslash G^{-1}\left(B_{\eta}^{p}\right) \rightarrow S_{1}^{p-1}$ is a proper map, and by using (6).

Finally, the fibrations (10) and (12) may be glued together along the fibration (11) to induce the locally trivial smooth fibration

$$
\Psi_{G \mid}: S_{\varepsilon}^{m-1} \backslash G^{-1}(\operatorname{Disc} G) \rightarrow S_{1}^{p-1} \backslash \operatorname{Disc} G
$$

which is independent of the small enough $\varepsilon>0$.

REMARK 3.7. One would like to have a more general existence result for a sphere fibration, namely without the radiality condition, like one can prove for isolated singularities [Mi] and more generally for a point discriminant [ACT, Theorem 2.1]. In order to do that, we need a blow-away vector field which is tangent to $\operatorname{Sing} G$, but one does not ask anymore that the vector field is tangent to the fibres of $\Psi_{G}$ (like we ought to do here, see $\S 4$ ). We conjecture that this can be done for any $\operatorname{Sing} G$ :

Conjecture 3.8. Under the conditions of Theorem 3.6 but without the radiality of the discriminant, there is a singular open book structure on the sphere $S_{\varepsilon}^{m-1} \backslash G^{-1}(\operatorname{Disc} G)$, independent of the small enough $\varepsilon$. 
ExAmple 3.9. Let $G: \mathbb{R}^{n} \rightarrow \mathbb{R}^{2}, G\left(x_{1}, \ldots, x_{n}\right)=\left(x_{1}, x_{2}^{2}+\cdots+x_{n-1}^{2}-x_{n}^{2}\right)$. One has $V_{G}=\left\{x_{1}=0\right\} \cap\left\{x_{2}^{2}+\cdots+x_{n-1}^{2}-x_{n}^{2}=0\right\}$ and $\operatorname{Sing} G=\left\{x_{2}=\cdots=x_{n}=0\right\}$, thus Disc $G=\mathbb{R} \times\{0\}$ is radial, and the map germ $G$ is nice since it verifies [ART, Lemma 2.4] quoted in $\S 2.1$. See also Example 3.3 for an alternate argument.

Since $M(G) \cap V_{G} \subset$ Sing $G \cap V_{G}=\{0\}$, the condition (4) is satisfied. By straightforward computations one gets that $M(\Psi)=\operatorname{Sing} G$, thus $M(\Psi) \backslash G^{-1}(\operatorname{Disc}(G))=\emptyset$, thus $\Psi$ is $\rho$-regular. By Theorem 3.6 it follows that $G$ has a Milnor-Hamm sphere fibration.

\section{Equivalence of Milnor-Hamm tube And sphere fibrations}

Let $G:\left(\mathbb{R}^{m}, 0\right) \rightarrow\left(\mathbb{R}^{p}, 0\right), m>p \geq 2$, be an analytic nice map germ.

Problem 4.1 (The Equivalence Problem). Assuming that the Milnor-Hamm tube fibration and sphere fibration exist, under what conditions are they equivalent, in the sense that the fibrations (3) and (6) are equivalent?

The equivalence problem 4.1 makes sense in the case of a radial discriminant since the bases of the fibrations (3) and (6) can be identified via multiplication by $\eta$, as we have explained in last part of Step 1 of the proof of Theorem 3.6.

Milnor [Mi] introduced the method of "blowing away the tube to the sphere" which uses integration of a special vector field in order to prove the equivalence of these two fibrations in the case of non-constant holomorphic function germs $\left(\mathbb{C}^{n}, 0\right) \rightarrow(\mathbb{C}, 0)$. As pointed out by Milnor [Mi] and explained in all details in [ACT, §2], this method may be applied in the real setting under certain conditions, still referring to a point-discriminant. We now formulate the properties of such a vector field in the more general context of a radial discriminant Disc $G$ :

Definition 4.2. One calls Milnor vector field for $G$ a vector field $\nu$ which satisfies the following conditions for any $x \in B_{\varepsilon}^{m} \backslash G^{-1}(\operatorname{Disc} G)$ :

(c1) $\nu(x)$ is tangent to the fibre $\Psi_{G}^{-1}\left(\Psi_{G}(x)\right)$,

(c2) $\langle\nu(x), \nabla \rho(x)\rangle>0$

(c3) $\left\langle\nu(x), \nabla\|G(x)\|^{2}\right\rangle>0$.

We then have the following general equivalence theorem:

Theorem 4.3. Let $G:\left(\mathbb{R}^{m}, 0\right) \rightarrow\left(\mathbb{R}^{p}, 0\right), m>p \geq 2$ be an analytic nice map germ with radial discriminant, such that the Milnor-Hamm tube fibration (2) and sphere fibration (6) do exist. If there is a Milnor vector field for $G$, then the fibrations (3) and (6) are equivalent.

Proof. The proof follows Milnor's pattern [Mi] explained in detail in [ACT]. The Milnor vector field is by definition radial pointing to the exterior of the spheres. It is well-defined and non-zero by definition outside the inverse image of the discriminant, and the excepted set Disc $G$ is itself radial by assumption (Definition 3.1), thus it is a collection of radii in the target. Therefore the Milnor vector field produces a flow on $B_{\varepsilon}^{m} \backslash G^{-1}(\operatorname{Disc} G)$, the projection by $G$ of which is radial in the target.

Let us remark here that the flow does not cross the set $G^{-1}$ (Disc $G$ ) precisely because the vector field is tangent to the fibres of $\Psi_{G}$ (i.e. condition $(\mathrm{c} 1)$ ) and that the discriminant 
is radial. This flow yields an isotopy from the Milnor-Hamm tube fibration to the MilnorHamm sphere fibration.

In the real setting, the Milnor vector field existence problem appeared first in case $\operatorname{Sing} G=\{0\}$ in Jacquemart's work [Ja] and later in [Ar, Oka2] etc. The more general case $\operatorname{Disc} G=\{0\}$ has been addressed later in e.g. [AT1, AT2, Ar, ACT, Han, Oka3]. The authors produced sufficient conditions in each setting, but the existence of a Milnor vector field without conditions seems to be an open problem ${ }^{4}$.

Equivalence Conjecture 4.4. Let $G:\left(\mathbb{R}^{m}, 0\right) \rightarrow\left(\mathbb{R}^{p}, 0\right), m>p \geq 2$ be an analytic nice map germ with radial discriminant and such that $\Psi_{G}$ is $\rho$-regular. If both fibrations (2) and (6) exist, then the fibrations (3) and (6) are equivalent.

Let us remind that the complement $S_{\eta}^{p-1} \backslash \operatorname{Disc} G$ may have several connected components and thus two types of fibrations over each such component.

Definition 4.5. We say that the fibrations (3) and (6) are fibre-equivalent if the corresponding fibres over each connected component of $S_{\eta}^{p-1} \backslash$ Disc $G$ are isotopic.

We show here that Conjecture 4.4 is true at the level of fibres.

Theorem 4.6. Let $G=\left(G_{1}, \ldots, G_{p}\right):\left(\mathbb{R}^{m}, 0\right) \rightarrow\left(\mathbb{R}^{p}, 0\right)$ be an analytic nice map germ with radial discriminant such that $\Psi_{G}$ is $\rho$-regular. If the Milnor-Hamm fibrations (2) and (6) exist, then:

(a) the fibrations (3) and (6) are fibre-equivalent,

(b) the fibrations (3) and (6) are equivalent over any contractible component of $S^{p-1} \backslash$ $\operatorname{Disc} G$.

Proof of Theorem 4.6. Since (b) is a simple consequence of (a), we stick to the proof of (a). For any vector field $\omega$ on $B_{\varepsilon}^{m} \backslash G^{-1}(\operatorname{Disc} G)$, we denote by $\operatorname{proj}_{T}(\omega(x))$ the orthogonal projection of $\omega(x)$ to some linear subspace $T \subset T_{x} B_{\varepsilon}^{m}=\mathbb{R}^{m}$. Milnor [Mi] proved the following result by using the Curve Selection Lemma, see also [Io, Lemma 1, p. 343].

Lemma 4.7. [Mi] Let $X \subset \mathbb{R}^{m}$ be an analytic manifold such that $0 \in \bar{X}$. Let $f$ and $g$ be analytic functions on $\mathbb{R}^{m}$ such that $f(0)=g(0)=0$ and that $f_{\mid X \backslash\{0\}}>0$ and $g_{\mid X \backslash\{0\}}>0$. Then there exists $\varepsilon>0$ such that for all $x \in X \cap B_{\varepsilon}^{m}$ the vectors $\operatorname{proj}_{T_{x} X}(\nabla f(x))$ and $\operatorname{proj}_{T_{x} X}(\nabla g(x))$ cannot have opposite direction whenever both are non-zero.

We apply this lemma to the following situation. Let $X_{y}:=\Psi_{G}^{-1}(y)$ be the fibre over some fixed value $y \in S_{1}^{p-1} \backslash \operatorname{Disc} G$. We may assume, without loss of generality, that $x$ belongs to the open set $\left\{G_{1}(x) \neq 0\right\}$. By [AT1, Section 2] and [AT2, Proof of Theorem $2.2]$, the normal space $N_{x} X_{y}$ of the fibre $X_{y}$ in $\mathbb{R}^{m}$ is spanned by $\left\{\Omega_{2}(x), \ldots, \Omega_{p}(x)\right\}$ where $\Omega_{k}=G_{1} \nabla G_{k}-G_{k} \nabla G_{1}$, for $k=2, \ldots, p$.

\footnotetext{
${ }^{4}$ The existence proof of [CSS, Lemma 5.2] has been reported as being incomplete, see [Han, iv] and [Ri, §7.3].
} 
Consider the following vector fields on $B_{\varepsilon}^{m} \backslash G^{-1}(\operatorname{Disc} G)$ :

$$
\begin{aligned}
& v_{1}(x):=\operatorname{proj}_{T_{x} X_{y}}\left(\nabla\|G(x)\|^{2}\right) \\
& v_{2}(x):=\operatorname{proj}_{T_{x} X_{y}}(\nabla \rho(x)) .
\end{aligned}
$$

The vector field $v_{1}$ has no zeros since the tube $\|G(x)\|^{2}=$ const. is transversal to $X_{y}$, for $y \notin \operatorname{Disc} G$. The second vector field $v_{2}$ has no zeros on $B_{\varepsilon}^{m} \backslash G^{-1}$ (Disc $G$ ) if and only if $M\left(\Psi_{G}\right) \backslash G^{-1}(\operatorname{Disc} G)=\emptyset$, i.e. $\Psi_{G}$ is $\rho$-regular, which is our assumption.

By Lemma 4.7, there is $\varepsilon>0$ such that for any $x \in X_{y} \cap B_{\varepsilon}$ either the vectors $v_{1}(x)$ and $v_{2}(x)$ are linearly independent, or they are linearly dependent but cannot have opposite direction.

Let $\varepsilon_{0}>0$ such that both fibrations (2) and (6) exist, for any $0<\varepsilon<\varepsilon_{0}$ and $0<\eta \ll$ $\varepsilon \leq \varepsilon_{0}$. Let us fix some $y \in S_{1}^{p-1} \backslash \operatorname{Disc} G$.

By the above Lemma and discussion, there is $0<\varepsilon<\varepsilon_{0}$ such that $v_{1}(x)$ and $v_{2}(x)$ cannot have strictly opposite direction on $B_{\varepsilon}^{m} \cap X_{y}$. Using Milnor's original idea, we may consider the bisector vector field:

$$
\nu(x)=\frac{v_{1}(x)}{\left\|v_{1}(x)\right\|}+\frac{v_{2}(x)}{\left\|v_{2}(x)\right\|}
$$

defined on $B_{\varepsilon}^{m} \backslash G^{-1}(\operatorname{Disc} G)$. It has no zeros on $X_{y} \cap\left(B_{\varepsilon}^{m} \backslash G^{-1}(\operatorname{Disc} G)\right)$ precisely because $v_{1}$ and $v_{2}$ do not point in opposite directions. This is thus a Milnor vector field on $B_{\varepsilon}^{m} \cap X_{y}$ and we may now apply Theorem 4.3 to the restriction of $G$ to the space $B_{\varepsilon}^{m} \cap X_{y}$ in order to prove the isotopy of the fibres over $y$ of the two fibrations.

Varying the point $y \in S_{1}^{p-1} \backslash \operatorname{Disc} G$ we get the isotopy of the corresponding fibres over any connected component.

4.1. The importance of the Milnor set $M(G)$. We tacitly assume throughout this subsection that $G$ is an analytic nice map with radial discriminant and that the MilnorHamm fibrations (2) and (6) exist. Let us show that the obstruction to the existence of a Milnor vector field is the Milnor set.

Lemma 4.8. Let $x \in B_{\varepsilon} \backslash G^{-1}(\operatorname{Disc} G)$. The vectors $v_{1}$ and $v_{2}$ are linearly dependent if and only if $x \in M(G)$. In particular the vector field $\nu$ from (13) is a Milnor vector field on $B_{\varepsilon}^{m} \backslash\left(M(G) \cup G^{-1}(\operatorname{Disc} G)\right)$.

Proof. For $x \in B_{\varepsilon} \backslash G^{-1}(\operatorname{Disc} G)$ we consider the decomposition:

$$
T_{x} X_{y}=T_{x} G^{-1}(G(x)) \oplus \mathbb{R}\left\langle v_{1}(x)\right\rangle,
$$

where, by its definition, the vector $v_{1}(x)$ is orthogonal to $T_{x} G^{-1}(G(x))$ in $T_{x} X_{y}$.

One writes $v_{1}(x)=v_{1}^{1}(x)+v_{1}^{2}(x)$ and $v_{2}(x)=v_{2}^{1}(x)+v_{2}^{2}(x)$ according to the decomposition (14). From the definitions, we have $v_{1}^{1}(x)=0$ for all $x \in B_{\varepsilon} \backslash G^{-1}(\operatorname{Disc} G)$, and $v_{2}^{1}(x)=0$ if and only if $x \in M(G)$. This proves the first claim, and the second is an easy consequence. 
For $x \in M(G) \backslash G^{-1}(\operatorname{Disc} G)$ one has that $v_{1}(x)$ and $v_{2}(x)$ are collinear, which amounts to the relation:

$$
\nabla \rho(x)=a(x) \nabla\|G(x)\|^{2}+\sum_{j=2}^{p} b_{j}(x) \Omega_{j}(x),
$$

where

$$
a(x)=\frac{\left\langle\nabla \rho(x), v_{1}(x)\right\rangle}{\left\|v_{1}(x)\right\|^{2}} .
$$

This proves in particular that $x \in M\left(\Psi_{G}\right) \backslash G^{-1}(\operatorname{Disc} G) \Leftrightarrow a(x)=0$.

With these notations one may characterise the existence of a Milnor vector field for $G$ as follows, extending the particular case Disc $G=\{0\}$ of [Han, Theorem 3.3.1].

Theorem 4.9. Let $G:\left(\mathbb{R}^{m}, 0\right) \rightarrow\left(\mathbb{R}^{p}, 0\right), m \geq p \geq 2$ be an analytic nice map with radial discriminant such that the Milnor-Hamm fibrations (2) and (6) exist. There exists a Milnor vector field for $G$ on $B_{\varepsilon} \backslash G^{-1}(\operatorname{Disc} G)$, for some small enough $\varepsilon>0$ if and only if $a(x)>0$ for any $x \in M(G) \backslash G^{-1}(\operatorname{Disc} G)$.

Proof. The implication " $\Rightarrow$ " follows from the definition (13) of the vector field $\nu(x)$. In fact, by condition (c1) one has that $\left\langle\nu(x), \Omega_{j}(x)\right\rangle=0$ for any $j=2, \ldots, p$. Therefore, $\langle\nabla \rho(x), \nu(x)\rangle=a(x)\left\langle\nabla\|G(x)\|^{2}, \nu(x)\right\rangle$, which by (c2) and (c3) implies that $a(x)>0$.

Reciprocally, if $a(x)>0$, it follows from Lemma 4.8 and (15) that the vector field $\nu(x)$ has no zeroes on $B_{\varepsilon}^{m} \backslash V_{G}$, hence it is a Milnor vector field for $G$.

There are several other criteria for the existence of a Milnor vector field for $G$; we discuss some of them in $[\mathrm{AR}]$.

Proposition 4.10. The image of the restriction $G: B_{\varepsilon}^{m} \cap M(G) \backslash G^{-1}(\operatorname{Disc} G) \rightarrow \mathbb{R}^{p}$ contains $B_{\eta}^{p} \cap \operatorname{Im} G \backslash \operatorname{Disc} G$ for some small enough ball $B_{\eta}^{p}$ centred at the origin.

Proof. Let $y \in B_{\eta}^{p} \cap \operatorname{Im} G \backslash \operatorname{Disc} G$, for some $\eta>0$ which fits in the Milnor-Hamm tube fibration, and such that the fibre $G^{-1}(y)$ is not empty. The distance function to the origin $\rho_{\mid}: \overline{B_{\varepsilon}^{m}} \cap \overline{G^{-1}(y)} \rightarrow \mathbb{R}_{\geq 0}$ has at least one local minimum point $x_{y}$ in the interior $B_{\varepsilon}^{m} \cap G^{-1}(y)$. This implies that $x_{y} \in M(G)$.

Let $\bigcup_{\beta} M_{\beta}$ be the decomposition into connected components of the subanalytic set germ at the origin $M(G) \backslash G^{-1}(\operatorname{Disc} G)$. See Example 5.4 where we have 8 connected components.

Corollary 4.11. If one of the following conditions holds:

(a) $M(G) \backslash G^{-1}(\operatorname{Disc} G)$ is connected,

(b) for any $\beta$ there is $y=y_{\beta} \in S_{1}^{p-1} \backslash \operatorname{Disc} G$ such that the germ at 0 of $M_{\beta} \cap X_{y}$ has dimension $>0$,

then there exists a Milnor vector field for $G$.

Proof. (a). Proposition 4.10 implies that $\operatorname{dim} M(G) \cap X_{y}>0$ for any $y \in S_{1}^{p-1} \backslash \operatorname{Disc} G$, thus (a) is a particular case of (b). 
(b). For some fixed $\beta$ we apply Milnor's Lemma 4.7 to that $X_{y}$ for which $\operatorname{dim} M_{\beta} \cap X_{y}>0$. This shows that the vectors $v_{1}(x)$ and $v_{2}(x)$ point in the same direction for all $x \in M_{\beta} \cap X_{y}$ and hence for all $x \in M_{\beta}$ since this is connected. Since this is true for any $\beta$ it is then true for the whole set $M(G) \backslash G^{-1}$ (Disc $G$ ), thus the vector field $\nu$ has no zeroes on it. Finally we may apply Lemma 4.8 to conclude that $\nu$ is a Milnor vector field.

\section{Classes of maps With equivalent Milnor-Hamm fibrations}

\subsection{Mixed functions and the equivalence problem.}

Definition 5.1. The mixed function $F: \mathbb{C}^{n} \rightarrow \mathbb{C}$ is called polar weighted-homogeneous of degree $k$ if there are non-zero integers $p_{1}, \ldots, p_{n}$ and $k>0$, such that $\operatorname{gcd}\left(p_{1}, \ldots p_{n}\right)=1$ and $\sum_{j=1}^{n} p_{j}\left(\nu_{j}-\mu_{j}\right)=k$, for any monomial of the expansion $F(\mathbf{z})=F(\mathbf{z}, \overline{\mathbf{z}})=\sum_{\nu, \mu} c_{\nu, \mu} \mathbf{z}^{\nu} \overline{\mathbf{z}}^{\mu}$.

The corresponding $S^{1}$-action on $\mathbb{C}^{n}$ is, for $\lambda \in S^{1}$ :

$$
\lambda \cdot(\mathbf{z}, \overline{\mathbf{z}})=\left(\lambda^{p_{1}} z_{1}, \ldots, \lambda^{p_{n}} z_{n}, \lambda^{-p_{1}} \bar{z}_{1}, \ldots, \lambda^{-p_{n}} \bar{z}_{n}\right) .
$$

Theorem 5.2. Let $F: \mathbb{C}^{n} \rightarrow \mathbb{C}$ be a polar weighted homogeneous mixed function. Then $F$ is a nice map germ with $\operatorname{Disc} F=\{0\}$, the Milnor fibrations (2) and (6) exist, and the fibrations (3) and (6) are equivalent.

Proof. From [ACT, §4.1] it follows (due to the $S^{1}$-action) that polar weighted-homogeneous maps are nice, more precisely because the image $F\left(B_{\varepsilon}^{2 n}\right)$ contains a small neighbourhood of the origin for any $\varepsilon>0$, and necessarily $\operatorname{Disc} F=\{0\}$. The discriminant is thus trivially radial.

It was proved in [PT1, Theorem 5.2] that a polar weighted-homogeneous mixed function germ $F$ has tube fibration, and in [ACT, Theorem 1.4] that such $F$ has a sphere fibration. It therefore remains to show that they are equivalent.

Each component $M_{\beta}$ of the decomposition into connected components $\bigcup_{\beta} M_{\beta}$ of the semi-analytic set germ at the origin $M(F) \backslash V_{F}$ is invariant under the $S^{1}$-action, more precisely one has that $\lambda M_{\beta}=M_{\beta}$ for any $\lambda \in S^{1}$. It follows that this verifies the hypothesis (b) of Corollary 4.11 and thus our claim follows.

5.2. Maps with radial action. Let $t \cdot x:=\left(t^{q_{1}} x_{1}, \ldots, t^{q_{m}} x_{m}\right)$ for $t \in \mathbb{R}_{+}$and $q_{1}, \ldots, q_{m} \in$ $\mathbb{N}^{*}$ relatively prime positive integers. One says that the map $G=\left(G_{1}, \cdots, G_{p}\right): \mathbb{R}^{m} \rightarrow \mathbb{R}^{p}$ is radial weighted-homogeneous (or radial, for short) of weights $\left(q_{1}, \ldots, q_{m}\right)$ and of degree $d>0$, if $G(t \cdot x)=t^{d} G(x)$.

Theorem 5.3. Let $G:\left(\mathbb{R}^{m}, 0\right) \rightarrow\left(\mathbb{R}^{p}, 0\right)$ be a radial weighted homogeneous map germ, and satisfying the condition (4). Then $G$ is nice, with radial discriminant, has MilnorHamm tube and sphere fibrations, and the fibrations (3) and (6) are equivalent.

Proof. The image of $G$ is a real cone and this cone is stable as a germ, in the sense that $G\left(B_{\varepsilon}^{m}\right)$ and $G\left(B_{\varepsilon^{\prime}}^{m}\right)$ have the same germs at the origin, for any $0<\varepsilon^{\prime}<\varepsilon$. Moreover, the boundary $\partial \overline{\operatorname{Im} G}$ is also a conical set germ at the origin.

The image by $\mathrm{G}$ of any analytic germ $X \subset \mathbb{R}^{m}$ which is invariant under the $\mathbb{R}_{+}$-action is a conical germ, and $\operatorname{Sing} G$ is such an invariant set germ. It follows that Disc $G$ is 
well-defined as a germ, and it is radial. These show that $G$ is a nice map germ, without using the hypothesis about condition (4).

The assumed condition (4) insures now the existence of the Milnor-Hamm tube fibration via [ART, Lemma 3.3]. Let us see that the Milnor-Hamm sphere fibration exists too. It was proved in [ACT, Prop. 3.2] by using the Euler vector field $\gamma(x):=\sum_{j=1}^{m} q_{j} x_{j}\left(\partial / \partial x_{j}\right)$ that the spheres are transversal to the fibres of the map $\Psi_{G}$. In our setting this implies that $\Psi_{G}$ is $\rho$-regular, thus our claim follows by Theorem 3.6.

The existence of a Milnor vector field follows by noting that any connected component $M_{\beta}$ of $M(F) \backslash V_{F}$ is also invariant under the $\mathbb{R}_{+}$-action, and thus we may apply Corollary 4.11(b).

ExAmple 5.4. [ART, Example 5.6] Let $G:\left(\mathbb{R}^{3}, 0\right) \rightarrow\left(\mathbb{R}^{2}, 0\right)$ given by $G(x, y, z)=\left(x y, z^{2}\right)$ is radial homogeneous. One has $V_{G}=\{x=z=0\} \cup\{y=z=0\}$, Sing $G=\{z=0\} \cup\{x=$ $y=0\}$, Disc $G=\{(0, \beta) \mid \beta \geq 0\} \cup\{(\lambda, 0) \mid \lambda \in \mathbb{R}\}$, and $G^{-1}(\operatorname{Disc} G)=\{x=0\} \cup\{y=$ $0\} \cup\{z=0\}$. We see that $\operatorname{Disc} G$ is radial, as predicted by Theorem 5.3.

By further computations one gets $M(G)=\{x= \pm y\} \cup\{z=0\}$. To check that $G$ satisfies the condition (4), let us consider $p_{0}=\left(x_{0}, y_{0}, z_{0}\right) \in \overline{M(G) \backslash G^{-1}(\operatorname{Disc} G)} \cap V_{G}$. Then there is a sequence $p_{n}:=\left(x_{n}, y_{n}, z_{n}\right) \in M(G) \backslash G^{-1}(\operatorname{Disc} G)$ such that $p_{n} \rightarrow p_{0}$ with $p_{0} \in V_{G}$. Consequently, $z_{0}=0$ and $x_{n}= \pm y_{n} \neq 0$ since $p_{n} \notin G^{-1}(\operatorname{Disc} G)$. Thus $x_{0}=\lim x_{n}= \pm \lim y_{n}=y_{0}=0$, and therefore $p_{0}=(0,0,0)$.

Then by Theorem 5.3 the map germ $G$ has Milnor-Hamm tube and sphere fibration, and the fibrations (3) and (6) are equivalent.

Corollary 5.5. Let $(f, g)$ be a holomorphic map germ which is Thom regular at $V_{(f, g)}$, and such that $f$ and $g$ do not have common factor of order $>0$.

If $f \bar{g}$ is a radial weighted homogeneous function, then $f \bar{g}$ has Milnor-Hamm tube and sphere fibrations, and the fibrations (3) and (6) are equivalent.

Proof. The Thom regularity of $(f, g)$ implies the Thom regularity of $f \bar{g}$ by [ART, Theorem 4.3] which extends [PT1, Theorem 3.1], and thus condition (4) is verified and we may apply the above Theorem 5.3 to conclude.

Corollary 5.6. Let $f$ and $g$ be holomorphic, radial weighted-homogeneous such that the map germ $(f, g)$ is an ICIS. Then the map germ $f \bar{g}:\left(\mathbb{C}^{n}, 0\right) \rightarrow(\mathbb{C}, 0)$ has Milnor-Hamm tube and sphere fibrations, and the fibrations (3) and (6) are equivalent.

Proof. Since $(f, g)$ is an ICIS, it follows by [ART, Theorem 2.7] that the map germ $f \bar{g}$ is a nice map germ, and that it is Thom regular and has a Milnor-Hamm tube fibration, by [ART, Theorem 4.3(a)]. If we add up the $\mathbb{R}_{+}$-action then we get, as in Theorem 5.3 above, the existence of a Milnor-Hamm sphere fibration and thus the equivalence of the fibrations.

Let us point out that if $(f, g)$ is an ICIS then its discriminant Disc $f \bar{g}$ is either a union of semi-analytic curves or it is $\{0\}$. This is actually true for any complex analytic map germ $(f, g)$, as shown in [PT2, Theorem 2.3] and in [Oka4], together with precise criteria for Disc $f \bar{g}$ being positive dimensional. 
ExAmple 5.7. Let us consider the map $f \bar{g}$, where $f, g:\left(\mathbb{C}^{2}, 0\right) \rightarrow(\mathbb{C}, 0), f(x, y)=x^{2}+y^{2}$ and $g(x, y)=x^{2}-y^{2}$. One easily computes that Disc $f \bar{g}$ is the real axis in $\mathbb{C}$ (see e.g. [PT1, PT2]). This verifies the assumptions of Corollary 5.6, thus $f \bar{g}$ has Milnor-Hamm tube and sphere fibrations, and they are equivalent.

\section{REFERENCES}

[Ar] R.N. Araújo dos Santos, Equivalence of real Milnor fibrations for quasi-homogeneous singularities. Rocky Mountain J. Math. 42 (2012), no. 2, 439-449.

[ACT] R.N. Araújo dos Santos, Y. Chen, M. Tibăr, Singular open book structures from real mappings, Cent. Eur. J. Math. 11 (2013) no. 5, 817-828.

[ART] R.N. Araújo dos Santos, M.F. Ribeiro, M. Tibăr, Fibrations of highly singular map germs, Bull. Sci. Math., 155 (2019), 92-111.

[AR] R.N. Araújo dos Santos, M.F. Ribeiro, Geometrical conditions for the existence of a Milnor vector field, arXiv:1807.08206

[AT1] R.N. Araújo dos Santos, M. Tibăr, Real map germs and higher open books, arXiv:0801.3328.

[AT2] R.N. Araújo dos Santos, M. Tibăr, Real map germs and higher open book structures, 177-185. Geom. Dedicata 147 (2010), 177-185.

[BM] E. Bierstone, P.D. Milman, Semianalytic and subanalytic sets. Inst. Hautes Études Sci. Publ. Math. 67 (1988), 5-42.

[Ch] Y. Chen, Milnor fibration at infinity for mixed polynomials, Cent. Eur. J. Math. 12 (2014), no. $1,28-38$.

[CT] Y. Chen, M. Tibăr, Bifurcation values and monodromy of mixed polynomials, Math. Res. Lett., 2012, 19(1), 59-79.

[CSS] J.L. Cisneros, J. Seade, J. Snoussi, Milnor fibrations and d-regularity for real analytic singularities, Internat. J. Math. 21 (2010), no. 4, 419-434.

[Han] N.B. Hansen, Milnor's Fibration Theorem for Real Singularities. Master Thesis, Faculty of Mathematics and Natural Sciences University of Oslo. 2014.

[Io] I.N. Iomdin, Certain properties of an isolated singularity of real polynomial mapping. Mat. Zametki 13 (1973), 565-572.

[Ja] A. Jacquemard, Fibrations de Milnor pour des applications réelles. Boll. Un. Mat. Ital. B (7) 3 (1989), no. 3, 591-600.

[JT1] C. Joiţa, M. Tibăr, Images of analytic map germs, arXiv:1810.05158.

[JT2] C. Joiţa, M. Tibăr, Images of analytic map germs and singular fibrations, European Journal of Mathematics 6 (2020), 888-904. arXiv:1904.07571.

[Ma] D.B. Massey, Real analytic Milnor fibrations and a strong Eojasiewicz inequality in Real and complex singularities, Real and complex singularities, 268-292, London Math. Soc. Lecture Note Ser., 380, Cambridge Univ. Press, Cambridge, 2010.

[Mi] J.W. Milnor, Singular points of complex hypersurfaces, Annals of Mathematics Studies, No. 61 Princeton University Press, Princeton, N.J.; University of Tokyo Press, Tokyo 1968.

[Oka1] M. Oka, Topology of polar weighted homogeneous hypersurface, Kodai Math. J. 31 (2008), no. 2, 163-182.

[Oka2] M. Oka, Non degenerate mixed functions. Kodai Math. J. 33 (2010), no. 1, 1-62.

[Oka3] M. Oka, On Milnor fibrations of mixed functions, $a_{f}$-condition and boundary stability. Kodai Math. J. 38 (2015), no. 3, 581-603.

[Oka4] M. Oka, On the Milnor fibration for $f(z) \bar{g}(z)$, arXiv:1812.10909v2.

[PT1] A.J. Parameswaran, M. Tibăr, Thom regularity and Milnor tube fibrations, Bull. Sci. Math. 143 (2018), 58-72.

[PT2] A.J. Parameswaran, M. Tibăr, Corrigendum to "Thom irregularity and Milnor tube fibrations" [Bull. Sci. Math. 143 (2018), 58-72], Bull. Sci. Math. 153 (2019), 120-123. 
[Ri] M.F. Ribeiro, Singular Milnor Fibrations. PhD Thesis, Instituto de Ciências Matemáticas e de Computação, São Carlos. University of São Paulo, February 2018. doi:10.11606/T.55.2018.tde-06072018-115031, http://www.teses.usp.br/teses/disponiveis/55/55135/tde-06072018-115031/en.php

[RA] M.A.S. Ruas, R.N. Araújo dos Santos, Real Milnor Fibrations and (C)-regularity. Manuscripta Math. 117 (2005), no. 2, 207-218.

[RSV] M.A.S. Ruas, J. Seade, A. Verjovsky, On Real Singularities with a Milnor Fibrations, Trends in singularities, 191-213, Trends Math., Birkhäuser, Basel, 2002.

[Ti] M. Tibăr, Regularity of real mappings and non-isolated singularities. in: Topology of Real Singularities and Motivic Aspects. Oberwolfach Rep. 9 (2012), no. 4, 2933-2935.

ICMC, Universidade de São Paulo, Av. Trabalhador São-Carlense, 400 - CP Box 668, 13560-970 São Carlos, São Paulo, Brazil

E-mail address: rnonato@icmc.usp.br, raimundo.icmc@gmail.com

Universidade Federal do Espirito Santo, Av. Fernando Ferrari, 514 - Goiabeiras - CP 29.075-910 Vitória, EsPirito SANTO, BraziL

E-mail address: maico.ribeiro@ufes.br

Univ. Lille, CNRS, UMR 8524 - Laboratoire Paul Painlevé, F-59000 Lille, France

E-mail address: mihai-marius.tibar@univ-lille.fr 